\title{
How Primary Care Providers Talk to Patients about Genome Sequencing Results: Risk, Rationale, and Recommendation
}

\author{
Jason L. Vassy, MD, MPH, $S M^{1,2,3}$, J. Kelly Davis, $B A^{4}$, Christine Kirby, $M A^{4}$, lan J. Richardson, $B A^{2}$, \\ Robert C. Green, MD, MPH $H^{3,5,6}$, Amy L. McGuire, JD, PhD ${ }^{7}$, and Peter A. Ubel, MD ${ }^{4,8}$
}

\begin{abstract}
'Section of General Internal Medicine, VA Boston Healthcare System, Boston, MA, USA; 2 Division of General Internal Medicine and Primary Care, Brigham and Women's Hospital, Boston, MA, USA; ${ }^{3}$ Department of Medicine, Harvard Medical School, Boston, MA, USA; ${ }^{4}$ Margolis Center for Health Policy, Duke University, Durham, NC, USA; ${ }^{5}$ Division of Genetics, Brigham and Women's Hospital, Boston, MA, USA; 'Broad Institute of MIT and Harvard, Cambridge, MA, USA; ${ }^{7}$ Center for Medical Ethics and Health Policy, Baylor College of Medicine, Houston, TX, USA; ${ }^{8}$ Fuqua School of Business, Sanford School of Public Policy, School of Medicine, Duke University, Durham, NC, USA.
\end{abstract}

BACKGROUND: Genomics will play an increasingly prominent role in clinical medicine.

OBJECTIVE: To describe how primary care physicians (PCPs) discuss and make clinical recommendations about genome sequencing results.

DESIGN: Qualitative analysis.

PARTICIPANTS: PCPs and their generally healthy patients undergoing genome sequencing.

APPROACH: Patients received clinical genome reports that included four categories of results: monogenic disease risk variants (if present), carrier status, five pharmacogenetics results, and polygenic risk estimates for eight cardiometabolic traits. Patients' office visits with their PCPs were audiorecorded, and summative content analysis was used to describe how PCPs discussed genomic results.

KEY RESULTS: For each genomic result discussed in 48 PCP-patient visits, we identified a "take-home" message (recommendation), categorized as continuing current management, further treatment, further evaluation, behavior change, remembering for future care, or sharing with family members. We analyzed how PCPs came to each recommendation by identifying 1) how they described the risk or importance of the given result and 2) the rationale they gave for translating that risk into a specific recommendation. Quantitative analysis showed that continuing current management was the most commonly coded recommendation across results overall (492/749, 66\%) and for each individual result type except monogenic disease risk results. Pharmacogenetics was the most common result type to prompt a recommendation to remember for future care $(94 / 119,79 \%)$; carrier status was the most common type prompting a recommendation to share with family members (45/54, 83\%); and polygenic results were the most common type prompting a behavior change recommendation $(55 / 58,95 \%)$. One-fifth of recommendation codes associated with monogenic results were for further evaluation $(6 / 24,25 \%)$. Rationales for these

Electronic supplementary material The online version of this article (https://doi.org/10.1007/s11606-017-4295-4) contains supplementary material, which is available to authorized users.

Received September 1, 2017

Revised November 14, 2017

Accepted December 20, 2017

Published online January 26, 2018 recommendations included patient context, family context, and scientific/clinical limitations of sequencing.

CONCLUSIONS: PCPs distinguish substantive differences among categories of genome sequencing results and use clinical judgment to justify continuing current management in generally healthy patients with genomic results.

KEY WORDS: genome sequencing; physician communication; medical decision-making.

J Gen Intern Med 33(6):877-85

DOI: $10.1007 / \mathrm{s} 11606-017-4295-4$

(c) Society of General Internal Medicine (outside the USA) 2018

\section{INTRODUCTION}

Individuals are increasingly learning about their genetic makeup. The use of genome sequencing, including whole-exome and whole-genome sequencing, continues to expand in clinical domains such as oncology, developmental delay, and prenatal medicine. ${ }^{1-6}$ Other people are learning more about their own genomes when their family members undergo sequencing. $^{7-9}$ Outside clinical care, some are learning about their genetic makeup through commercial genome sequencing services or participation in research. ${ }^{10}$ Tens of thousands of individuals have already received genome sequencing results through clinical or research channels, and programs including the 100,000 Genomes Project in the United Kingdom, ${ }^{11}$ the Precision Medicine Initiative All of Us Research Program in the United States, ${ }^{12}$ and others ${ }^{10,13-16}$ are poised to increase this number by millions in the near future.

As a result of these trends, primary care physicians (PCPs) will be increasingly faced with managing complex genome sequencing information with uncertain clinical significance. In addition, as medical specialists such as oncologists and neurologists increasingly order genome sequencing for specific clinical indications, they will more frequently identify secondary findings unrelated to the primary indication for sequencing. ${ }^{17,18}$ For example, a cardiologist might identify a BRCAl breast cancer variant during the evaluation of a patient's cardiomyopathy. Specialists might refer these patients back to their PCPs to 
coordinate the evaluation and management of such off-target results. ${ }^{19,20}$ Moreover, although risk communication and counseling about genetic results has traditionally been the domain of genetic counselors and medical geneticists, the insufficient numbers of these specialists ${ }^{21,22}$ may limit the availability of formal genetics consultation for all patients who undergo genome sequencing. Thus, PCPs may be called upon to fill these gaps, either formally when specialists refer patients back to them for management of sequencing results unrelated to their specialty, or informally when patients turn to them with followup questions about the results and their medical care. ${ }^{23}$

Genetics involves the analysis of specific and limited sets of genes, and might still be unfamiliar territory for many PCPs. The newer field of genomics brings additional complexity with its vastly larger scope and the different types of results that it can deliver (Table 1). Genome-wide tests include certain direct-to-consumer products such as those from 23 andMe, which might include only hundreds of thousands of common genomic markers. Genome-wide testing also includes genome sequencing, which, in contrast, can read almost all three billion base pairs comprising the human genome. ${ }^{24}$ As a result, genome sequencing can identify several categories of results, ${ }^{25}$ ranging from rare variants associated with significant monogenic diseases, to common risk alleles with small effect sizes for complex polygenic conditions like type 2 diabetes. For each person, genome sequencing might identify more than 50 rare variants that have never before been seen. ${ }^{26}$ Much uncertainty remains in interpreting the genome sequence of an individual, and these interpretations are subject to change over time as new scientific evidence accrues. ${ }^{24,27-29}$ We have previously reported that adding genome sequencing to primary care yields uncertain clinical value. ${ }^{27}$

Nonetheless, PCPs will be on the front lines of this uncertainty as genomics increasingly permeates clinical medicine. ${ }^{30}$
Little is known about how PCPs discuss genome sequencing results with their patients. ${ }^{31}$ Anticipating that $\mathrm{PCP}$-patient conversations about genomics will become more common in the future, we recruited PCPs and asked them to discuss genome sequencing results with their patients. Analyzing these clinical discussions, we sought to illuminate how PCPs communicate different types of genome sequencing results and their management recommendations for those results of uncertain clinical utility.

\section{METHODS}

\section{Study Design}

The MedSeq Project (Clinicaltrials.gov Identifier NCT01736566) is a pair of pilot trials of the integration of whole-genome sequencing in two clinical contexts: subspecialty cardiology and primary care. ${ }^{27,32}$ Here we report the experience of PCPs discussing genome sequencing results with their patients. In brief, 50 generally healthy adults underwent sequencing and received their results from their PCPs during audio-recorded office visits. We analyzed the transcripts of these clinical discussions. The Partners Human Research Committee approved this study.

\section{Participants}

We recruited nine PCPs from clinics at one academic medical center. ${ }^{27,32}$ Study staff recruited PCPs through presentations at clinic staff meetings and individual outreach. We previously reported that these PCPs were motivated to participate in the MedSeq Project by the opportunity to learn about genomics in a monitored environment ${ }^{19}$; although they were uncertain that genome sequencing was

Table 1 Four Categories of Genome Sequencing Results Reported to 48 Generally Healthy Patients

\begin{tabular}{|c|c|c|c|}
\hline Result category & Description & $\begin{array}{l}\text { Patients with } \\
\text { results, } n(\%)\end{array}$ & Examples \\
\hline $\begin{array}{l}\text { Monogenic disease } \\
\text { risk }\end{array}$ & $\begin{array}{l}\text { Result indicating risk of a rare Mendelian disease for } \\
\text { the patient him- or herself: a single variant associ- } \\
\text { ated with an autosomal dominant disease (or an X- } \\
\text { linked disease in a male) or two variants associated } \\
\text { with an autosomal recessive disease. Variants were } \\
\text { categorized as pathogenic (P), likely pathogenic } \\
\text { (LP), or variant of uncertain significance (VUS) }\end{array}$ & $\begin{array}{l}\text { P/LP: } 9(19) \\
\text { VUS: } 7(15)\end{array}$ & $\begin{array}{l}\text { Single LP variant in } K C N Q 1 \text { (Romano-Ward } \\
\text { syndrome, inherited in autosomal dominant } \\
\text { manner) } \\
\text { Two P variants in } R D H 5 \text { (fundus albipunctatus, } \\
\text { inherited in autosomal recessive manner) }\end{array}$ \\
\hline Carrier status & $\begin{array}{l}\text { Result indicating that the patient is a carrier of a rare } \\
\text { recessive Mendelian disease, usually indicating no } \\
\text { risk to the patient him- or herself: a single variant in } \\
\text { a gene associated with autosomal recessive disease, } \\
\text { categorized as P, LP, or VUS }\end{array}$ & $48(100)$ & $\begin{array}{l}\text { Single variant in CFTR (cystic fibrosis) } \\
\text { Single variant in } U S H 2 A \text { (Usher syndrome) }\end{array}$ \\
\hline Polygenic disease risk & $\begin{array}{l}\text { Quantitative risk estimates for } 8 \text { common } \\
\text { cardiometabolic traits: abdominal aortic aneurysm, } \\
\text { atrial fibrillation, coronary heart disease, } \\
\text { hypertension, obesity, platelet aggregation, QT } \\
\text { prolongation, type } 2 \text { diabetes }\end{array}$ & $48(100)$ & $\begin{array}{l}\text { Type } 2 \text { diabetes: } 5-10 \% \text { of variation is explained } \\
\text { by common genetic variants. This patient's } \\
\text { polygenic relative risk is } 1.4 \text { (percentile rank: } \\
60 \text { th-70th). }\end{array}$ \\
\hline $\begin{array}{l}\text { Pharmacogenetic } \\
\text { (PGx) association }\end{array}$ & $\begin{array}{l}\text { Drug response prediction for } 5 \text { common } \\
\text { medications: clopidogrel, digoxin, metformin, } \\
\text { simvastatin, warfarin }\end{array}$ & $48(100)$ & $\begin{array}{l}\text { Warfarin: Increased dose requirement } \\
\text { Simvastatin: Typical risk of myopathy }\end{array}$ \\
\hline
\end{tabular}

Italicized abbreviations refer to gene names 
currently useful for their practices, they generally expected its clinical utility to increase during their careers. ${ }^{33} \mathrm{We}$ have previously described the brief continuing medical education-eligible curriculum PCPs completed before enrolling patients, ${ }^{19,32}$ which included online case-based modules and two in-person sessions covering Mendelian inheritance patterns, risk prediction, genomics support resources, a general overview of genome sequencing, and an orientation to the study's genome report (Online Supplementary Figure 1). ${ }^{34}$ The curriculum did not include management recommendations for specific types of results or instructions for discussing results with patients. Participating PCPs helped identify and recruit approximately 10 of their patients until the predetermined sample size of 100 was reached. ${ }^{27}$ Eligible patients were aged $40-65$ years and were generally healthy in the PCPs' judgment.

\section{Genome Sequencing and Reporting}

Whole-genome sequencing was performed as described previously, and the Partners Laboratory for Molecular Medicine interpreted variants in more than 4600 genes associated with human disease. ${ }^{25}$ Each patient's PCP received an interpreted genome report (Online Supplementary Figure 1) that included different categories of genome sequencing results: 1) monogenic disease risk, 2) carrier status, 3) polygenic disease risk, and 4) pharmacogenetic (PGx) associations (Table 1). ${ }^{25,34,35}$ By design, all patients had eight polygenic risk estimates and five PGx results. The monogenic disease risk and carrier status sections of the report included variants categorized by accepted standards as pathogenic (P); likely pathogenic (LP); or, in certain cases, variant of uncertain significance (VUS). ${ }^{28}$ Most patients did not have a monogenic disease risk result, whereas all patients had at least one carrier status result (Table 1). The first page of the report summarized the findings from all result categories, while subsequent pages gave more detail about their interpretation, disease information, and familial risk. The genome report did not include recommendations for clinical management. ${ }^{34}$

\section{Study Procedures}

At the baseline visit, enrolled patients gave a blood sample and reported their family health history using an online tool. ${ }^{36}$ Patients were randomly assigned to receive only the pedigree from this family history tool or the combination of the pedigree and the genome report. Each patient's study reports (pedigree alone or pedigree plus genome report) were emailed to the PCP a few days before a scheduled disclosure visit, during which the PCP and patient met to discuss the reports. Disclosure visits were audio-recorded and transcribed verbatim.

\section{Analysis}

We restricted the present analyses to the discussions between PCPs and the half of patients receiving genome reports. The analytic team consisted of two qualitative researchers (JKD and CK) and two primary care physicians (JLV and PAU). We used summative content analysis consisting of a two-phase iterative process: inductive development of a coding scheme and deductive application of the coding scheme to all transcripts. ${ }^{37}$ After reading several transcripts individually, the analytic team met regularly over several months to discuss the thematic focus of how PCPs communicated and made recommendations about genome sequencing results to their patients.

Through this process, we inductively developed the conceptual model shown in Figure 1. We found that PCPs generally guided their patients through each section of the genome report in order, discussing each result, its potential health impact, and the PCP's recommendations, if any. For each individual genome sequencing result (e.g. a polygenic risk estimate for coronary artery disease or a carrier status for a rare autosomal recessive condition, biotinidase deficiency), we sought to identify the one or more take-home messages the PCP communicated to the patient about that result, which we coded as the recommendation(s). We then examined the explanation the PCP gave for each recommendation. We found that this

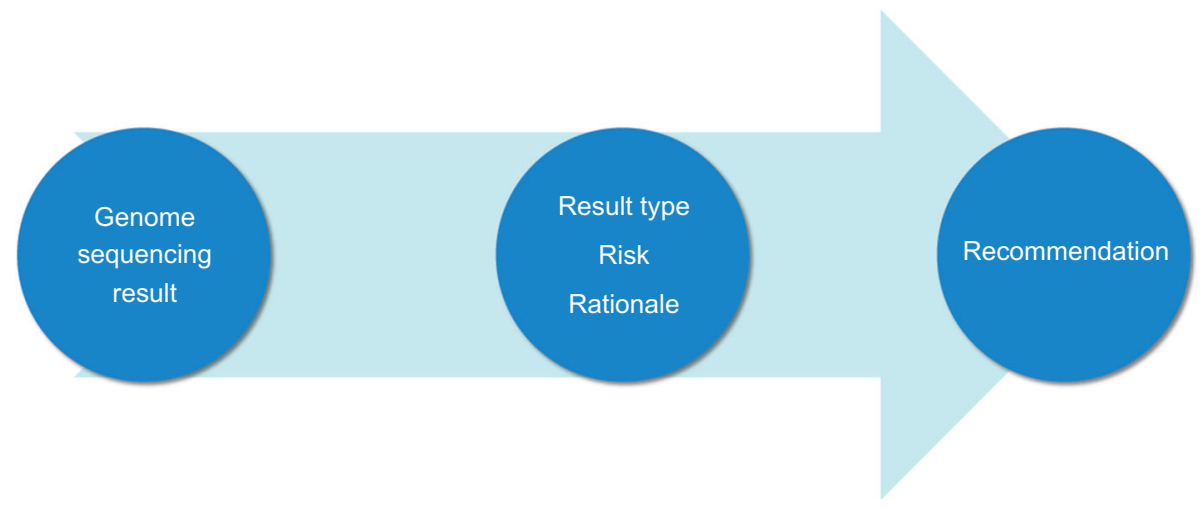

Figure 1 Conceptual framework for summative content analysis of PCP-patient discussions about genome sequencing results. For each result discussed, one or more take-home messages, or recommendations, were identified. The discussion about each recommendation was further coded by the type of result (e.g. monogenic disease risk, carrier status, polygenic risk, or pharmacogenetic result), the risk the PCP ascribed to that result, and the rationale(s) given for the recommendation. 
explanation often included a level of risk the PCP attributed to the result and one or more rationale(s) for why a sequencing result of that risk, contextualized with other information, led to the recommendation. For pharmacogenetic results, the risk dimension was more suitably called importance, as providers tended to speak more about the value of this information for medical care than about the likelihood of an associated disease. Using these three discrete dimensions of the conceptual model (risk/importance, rationale, and recommendation), the analytic team discussed several examples of $\mathrm{PCP}$-patient conversations until thematic saturation for codes in each dimension was reached. At this point, two qualitative analysts independently and deductively applied the coding scheme to all transcripts, using NVivo software, version 10 (QSR International Pty Ltd., Melbourne, Australia). Inter-rater agreement was excellent ( $\kappa$ range $0.75-1$, Online Supplementary Table 1). Within each coded transcript, we determined counts of codes within each of the three dimensions, stratified by the type of genome sequencing result discussed (monogenic disease risk, carrier status, polygenic disease risk, or pharmacogenetic).

Table 2 Example Quotes of Physician Descriptions, Explanations, and Recommendations Regarding Genome Sequencing Results

Risk/importance: How the physician describes the risk or importance of a specific genome sequencing result

No/low risk or importance

Uncertain risk or importance

Moderate risk or importance

High risk or importance

Rationale: The reasoning the physician gives for the assessment and take-home recommendation for each result Rationale not given or given in genetic terms only

Limitations: Clinical, scientific, or technical limitations of sequencing

Family context: Physician uses the health of patient's family members for contextualization

Patient context: Physician uses information about patient's medical history, family history, or lifestyle for contextualization

Recommendation: The physician's take-home message for each genome sequencing result

Relevant to family member

\section{Remember for future care}

Behavior change

Further evaluation

Further treatment

\section{Continue current management}

Q1. So they look at a whole bunch of different things and sort of make-talk about your risk of having certain things...So the risk of an abdominal aortic aneurysm - the [polygenic relative] risk was 0.9. Not a big deal. (Polygenic risk of abdominal aortic aneurysm)

Q2. This genetic variant suggests that you may be more susceptible to Cushing's syndrome; however...it's only been reported in one individual [in the world]. So... the clinical significance of this genetic variant is uncertain. (VUS in PDE11A, associated with primary pigmented nodular adrenocortical disease)

Q3. There is sort of this increased background risk. But having a healthy lifestyle is offsetting sort of whatever genetic increase risk of things that you have. (Polygenic risk of coronary artery disease, type 2 diabetes, and hypertension) Q4. [Your risk] is going high, you know? So it's almost three times the normal rate...So I think it does help give you some quantitative sense of your father's history of heart attack that your personal risk may be like three times. (Polygenic risk of coronary artery disease)

Q5. They say your risk is up to 2.8 compared to the normal population. (Polygenic risk of coronary artery disease)

Q6. Remember this - although this is looking at millions of combinations, it's missing about $5 \%$ to $10 \%$ of your total genome. So there may be other things that are there that we're not seeing.

Q7. So there's nothing I see in any of your tests that would change what I tell you all the time, which is to be careful about your diet, keep your cholesterol under control, exercise regularly. (Polygenic risk results)

Q8. But you do not need any of these medications at all, so it's not really relevant. But it is interesting, and it's good to have that information. (PGx results) Q9. If your wife was a carrier for the same gene, there would be a $25 \%$ chance that one of your children would have this illness. They would have had it by now... because it's something that manifests in childhood. (Carrier result for phenylketonuria)

Q10. This is a condition - if I can pronounce it correctly-called spondyloepiphyseal dysplasia congenita, which is really something that would manifest itself at birth. And as we can see, you are five foot ten, five foot eleven... I would not worry about this. (Likely pathogenic variant in COL2A1)

Q11. And this is something that you can pass. So this is less likely pathogenic for you, and it is that you can pass it on to your children. (Carrier variant in ERCC5, associated with xeroderma pigmentosum)

Q12. So, again, if you needed $a$-if you needed blood thinning for a certain reason, this is interesting to know, and they might want to put you on something else. So I'll definitely put this in your chart. (PGx result for clopidogrel) Q13. Type 2 diabetes - you are at increased risk. Obviously it's important to exercise and keep your weight down. (Polygenic risk of type 2 diabetes)

Q14. So what am I going to recommend about that? I am going to recommend that we get an EKG...And we actually have you set you up to see cardiology, and they can then do whatever stress tests or anything else they want to do. (Likely pathogenic variant in $K C N Q 1$, associated with Romano-Ward syndrome)

Q15. But if you believe this SNP association that says that you have a threefold increased risk of increased platelet aggregation, and given your family history of lots of coronary artery disease, I think that you would be a fair candidate for one baby aspirin a day. (Polygenic risk of platelet aggregation)

Q16. You have a slightly increased risk, 1.3. But if you do not have hypertension, I wouldn't worry too much about that. (Polygenic risk of hypertension)

Physician quotes $(Q)$ from 48 PCP-patient discussions about genome sequencing results, illustrating three dimensions of how physicians talk about each result. Parentheses indicate the specific result(s) discussed in the examples 


\section{RESULTS}

Transcripts from 48 PCP-patient conversations about genome sequencing results were available for analysis (Online Supplementary Table 2). The median length of the recordings was 31 (range 14-101) minutes.

\section{Thematic Codes and Frequencies}

Analysis identified six take-home recommendation codes: relevant to family member; remember for future care; need for behavior change; need for further evaluation; need for further treatment; and continue current management, a code that included both explicit recommendations not to change medical care and the absence of a recommendation (Table 2). Continue current management was the most commonly coded recommendation across all results overall (492 of 749 codes, $66 \%$ ) and for each individual result type except monogenic disease risk results. There were very few recommendations for further treatment. Rationale(s) for each recommendation were coded as: patient context, family context, scientific or clinical limitations, or rationale not given or given in genetic terms only (Table 2). Figure 2 shows the distribution of codes across the 48 transcripts, categorized by type of genome sequencing result. Because pathogenic (P) and likely pathogenic (LP) variants have greater scientific evidence supporting their role in human disease than variants of uncertain significance (VUS), we present these two categories of monogenic disease risk results (P/LP and VUS) separately.

\section{Combining Risk and Rationale in Making Recommendations}

Figure 3 represents the frequencies with which PCPs combined different levels of risk/importance assessment and $\mathrm{ra}$ tionales to generate recommendations for their patients, categorized by type of genomic result.

Physicians discussed pharmacogenetic results as having moderate $(189 / 229,83 \%)$ or high importance $(40 / 229$, $17 \%$ ). Despite this perceived importance, PCPs commonly discussed one clinical limitation of the information: that the patient did not currently need the medication in question $(\mathrm{Q} 8$ in Table 2). Pharmacogenetic results were the most common

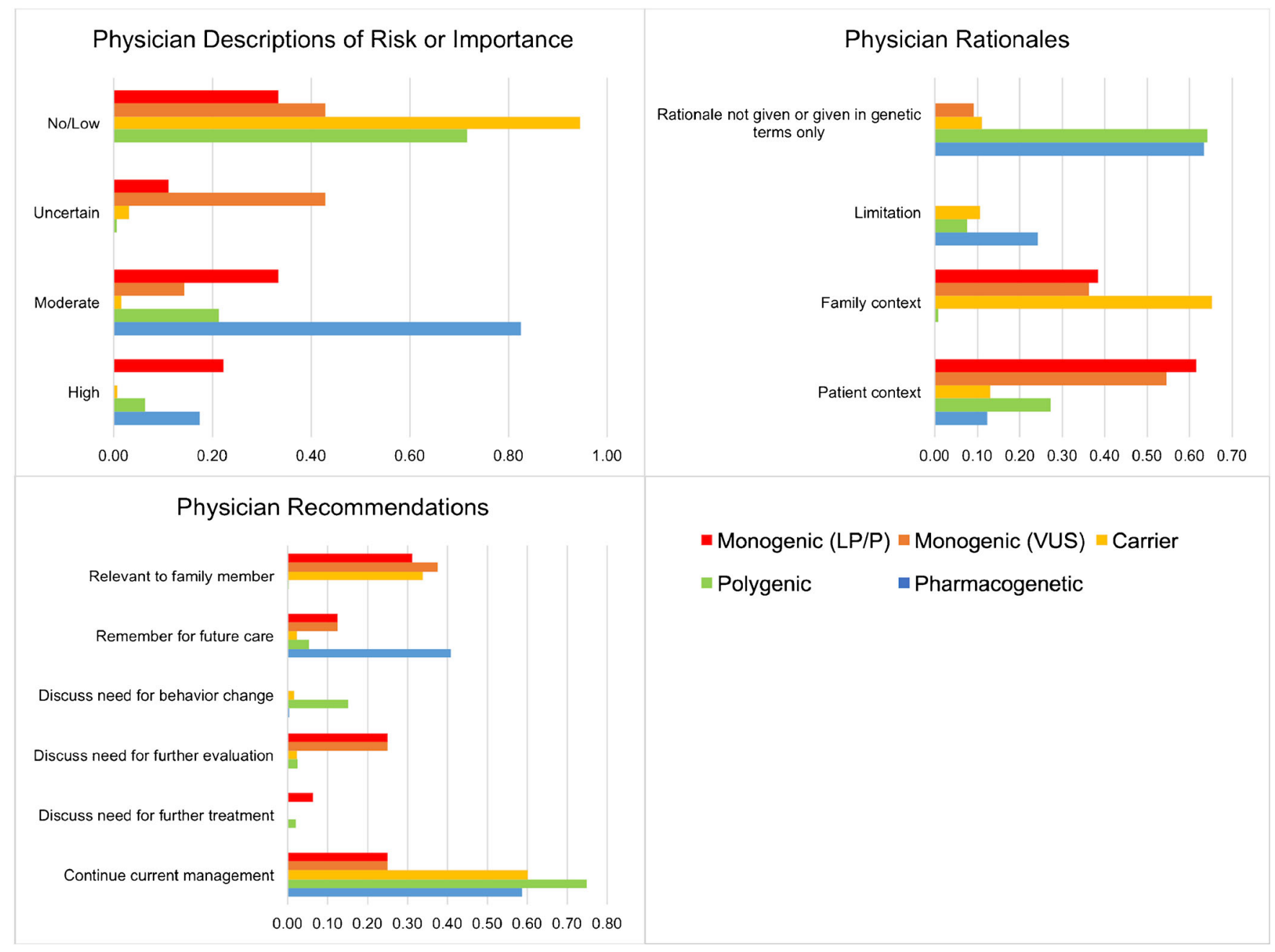

Figure 2 Proportions of codes in three distinct dimensions in 48 physician discussions about genome sequencing results: risk (or for pharmacogenetic results, importance), rationale, and recommendation. Codes are stratified into the five possible types of results, as shown in the legend. For some results, more than one rationale or recommendation code was identified. LP, likely pathogenic; P, pathogenic; VUS, variant of uncertain significance. 


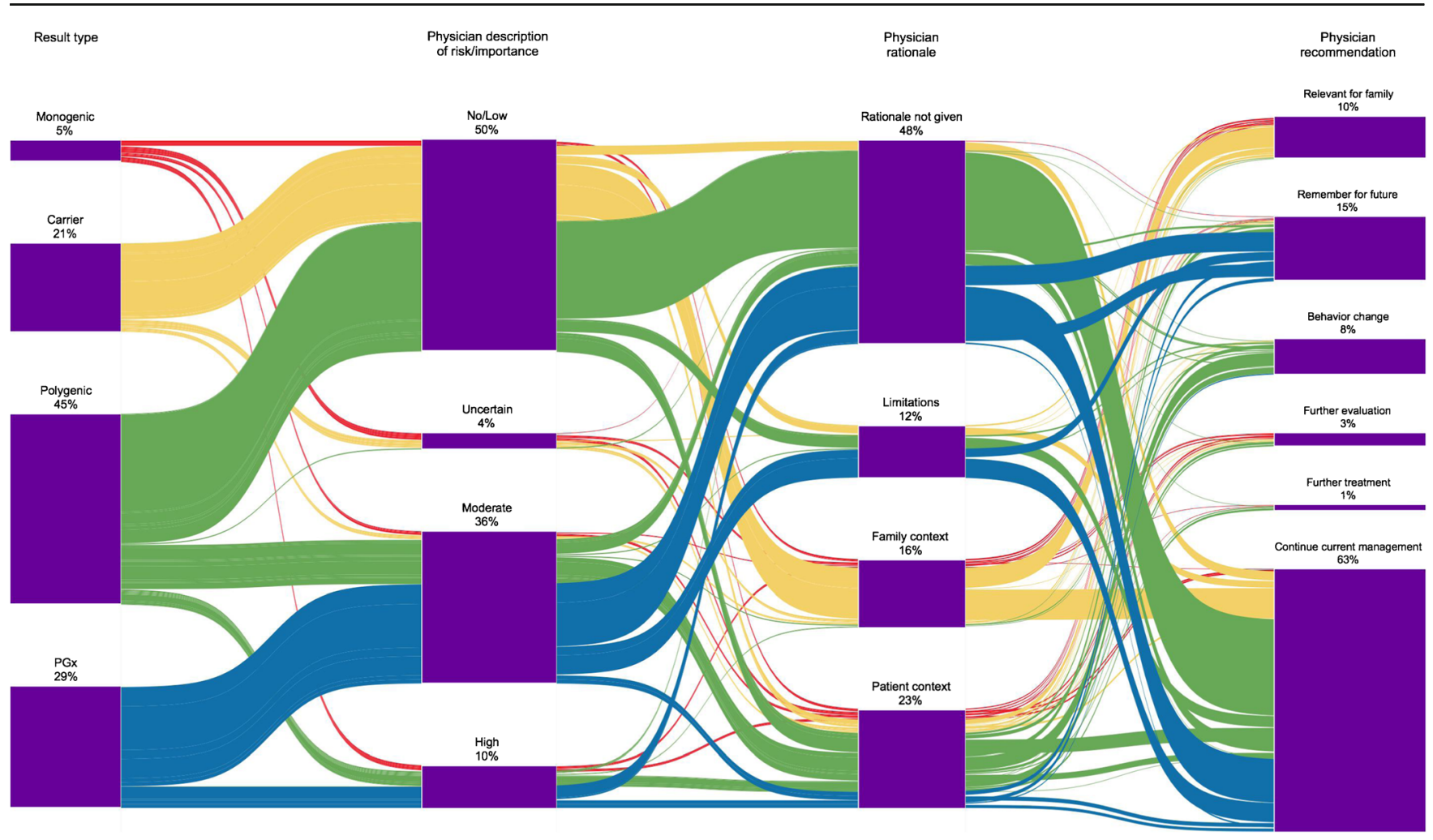

Figure 3 Sankey diagram mapping the combinations of risk/importance, rationale, and recommendation codes in physician discussions about genome sequencing results, presented by type of result. Percentages indicate the proportions of individual qualitative codes within each axis (risk/importance, rationale, and recommendation). The width of each pathway corresponds to the proportion of a given code combination (for example, a polygenic result described as uncertain risk, prompting a recommendation to continue current management, with no rationale given), relative to the total number of code combinations.

type associated with a recommendation code to remember for future care $(94 / 119,79 \%)$.

The majority of carrier status results were considered to be of no/low risk to the patient $(122 / 128,95 \%)$ and therefore not warranting change in current management. Family circumstances, such as the ages and health of any children, often helped PCPs contextualize the clinical significance of the results for patients (Q9). Carrier status was the most common result type associated with a relevant to family member code (45/54, 83\%), illustrated by Q11.

PCPs most often described polygenic risk estimates as having no/low risk (248/346, 72\%). Although we observed few behavior change recommendation codes in the transcripts overall $(58 / 749,8 \%)$, the majority $(55 / 58,95 \%)$ were associated with polygenic results. PCPs often gave patient context as a rationale when choosing between recommending a health behavior change (Q13) and continuing current management (Q7, Q16). PCPs also cited the limitations of genome sequencing technology as a reason not to act on the information (Q6).

Despite their smaller number, monogenic disease risk results were more evenly represented across all four categories in the risk dimension. PCPs used both patient and family context as rationales for continuing current management (Q10) or pursuing further evaluation (Q14). Unlike the other risk categories, $25 \%(6 / 24)$ of recommendation codes associated with monogenic results were for further evaluation.

\section{DISCUSSION}

Because of the expected growth in genome sequencing, ${ }^{10-16}$ PCPs will increasingly play a role in the management of their patients' genomic results. Despite this trend, there is concern that PCPs are not prepared to understand and use this inherently uncertain information in patient care. We identified two major findings in our analyses of 48 PCP-patient discussions about genome sequencing results of varying severity and clinical meaning. First, we observed that PCPs communicated different messages about different categories of sequencing results, such as carrier status and pharmacogenetics. Second, we observed a common tendency for PCPs to use contextual factors and the perceived limitations of genomic information to justify continuing current management, despite being confronted with a new result with potential clinical implications. This observation suggests that PCPs' clinical judgment might lessen the burden of unnecessary healthcare utilization in the face of uncertain genome sequencing results.

Several studies have reported that PCPs have limited experience with and knowledge of genetic testing, let alone genome sequencing, ${ }^{38-42}$ although this trend might be reversing. ${ }^{33,43-45}$ Early-adopter institutions are beginning to incorporate genomics education initiatives to improve providers' perceptions, ability, and use of genetics and genomics in clinical care. ${ }^{19,45-50}$ We previously reported that an external panel of geneticists determined that the PCPs in the MedSeq 
Project generally managed monogenic disease risk results appropriately. $^{27}$ The present analyses do not evaluate whether PCPs managed the other categories of results appropriately, but their patterns of communication about different categories of results suggest that they are able to distinguish substantive differences between the types of results genome sequencing can deliver. Reassuringly, these differences were generally consistent with how genetics professionals would interpret these categories of results. Specifically, carrier results were frequently identified as relevant to family members; PCPs frequently recommended remembering pharmacogenetic results for future care; and the category with the highest proportion of recommendations for further evaluation was the monogenic disease risk category. The clinical utility of polygenic risk estimates for common complex diseases remains unproven, particularly since they omit non-genetic contributors to disease such as smoking or obesity. ${ }^{44}$ That PCPs did not often recommend management changes due to polygenic results suggests that they appreciate this state of the science. These observations add to the growing body of literature demonstrating that PCPs, when provided with educational support, can learn enough about this new field to discuss uncertain genome sequencing results with their patients. ${ }^{44}$

We observed that the PCPs in this study adopted many of the communication techniques used by genetics professionals, reviewed recently by Paul and colleagues. ${ }^{51}$ Similar to observations of genetic counselors, ${ }^{56}$ PCPs in this study used individual patient and family histories to calibrate how they interpreted the risk of a given genomic result. When faced with genomic results that were seemingly discrepant from patient or family history, PCPs tended to reject the genomic information, either dismissing it outright or using hedging language to soften the rejection and convey the uncertainty in their interpretation and management. ${ }^{57,58}$ All this is not to say that PCPs discussed genome sequencing results flawlessly; we have previously cataloged difficulties they encountered with effective communication and with the volume and complexity of the information. ${ }^{52}$ However, like genetics professionals, they developed strategies to communicate the risk and uncertainty conveyed in genomic results ${ }^{53-55}$ and to incorporate those concepts into their medical decision-making. Uncertainty and the need for contextualization have always characterized the practice of medicine; while these observations may seem unnecessary to point out, they are a timely reminder as genomic initiatives are contemplating the large-scale return of results to potentially millions of individuals. Frontline clinicians will be a part of that process and are accustomed to handling the inherent uncertainty of medical information.

Our observations also highlight the importance of the format of a genome sequencing report in shaping how providers communicate and manage results. We observed that PCPs discussed the results in the order they appeared on the report, reading the results to patients and adding comments about interpretation and management. This pattern stems from the organization of the first page of the report, which included summary results clearly divided into separate sections for each result type. $^{25,34}$ Prior and ongoing work on optimizing how genomic test results are reported ${ }^{59-61}$ will be critical in ensuring that clinicians can manage them appropriately.

Finally, amid prevailing concerns that the increasing availability of genomic information will prompt additional medical management that is unnecessary and costly, ${ }^{62,63}$ we observed instances where PCPs' contextualization and clinical judgment guided them away from over-managing genomic results. Although a recent key informant study noted the technological and scientific limitations of genomics as potential safety concerns for genomic medicine, ${ }^{63}$ some PCPs in our study used these perceived limitations to justify inaction in the setting of certain genomic results. Deyo has described common situations in medical practice that can trigger unnecessary medical care, including erroneous interpretation of medical data and low tolerance for ambiguity. ${ }^{64}$ Although genomic medicine is susceptible to these conditions, the PCPs we observed exhibited mitigating strategies described by Deyo, namely, continuity of care and considering results in the full context of the patient's history and physical findings. ${ }^{64}$ As the genomic medicine evidence base grows, clinical guidelines and clinical decision support will further mitigate the risk of clinical overreaction to genomic results. ${ }^{65,66}$

The novelty and strength of this study lies in its analysis of audio recordings of actual clinical discussions between patients and their own PCPs about genome sequencing results. However, these analyses are limited to academic PCPs and their generally healthy patients participating in a research study. Moreover, PCPs completed a genomics educational curriculum that is not standard practice at present. As a result, our observations may not generalize to non-research and other diverse settings. Still, qualitative analysis aims not to make generalizable assertions, but to generate new themes and hypotheses from rich data. For example, the observation that PCPs often rationalized clinical inaction in managing a new genomic result merits further investigation.

In conclusion, although this study does not provide evidence to support the routine use of genome sequencing in primary care medicine, we observed that PCPs could distinguish different types of potentially clinically significant but inherently uncertain genome sequencing results in their healthy patients. Their use of contextual factors and the perceived limitations of genome sequencing information to justify continuing current management may be an asset serving to balance the competing risks of inappropriate under-management and over-management.

Contributors: The authors thank Mary Carol Barks, BA, and Sanjay Advani, MA, for assistance in preparing this manuscript.

Prior Presentations: Parts of this work were presented at the American Society for Human Genetics national meetings in 2014 and 2016.

Corresponding Author: Jason L. Vassy, MD, MPH, SM; Section of General Internal Medicine, VA Boston Healthcare System, Boston, MA, USA (e-mail: jvassy@partners.org). 
Funders The MedSeq Project is funded by grant U01-HG00650O from the National Human Genome Research Institute of the National Institutes of Health (NIH). Dr. Vassy is an employee of the VA Boston Healthcare System and received support from NIH grant KL2TROO110O and Career Development Award IK2-CXOO1262 from the VA Clinical Sciences Research and Development Service. Dr. Green is also supported by NIH U19-HD077671, U01-HG008685, R03HG008809, UG3-OD023156, U41-HG006834, UO1-AG24904, RO1CA154517, P60-AR047782, R01-AG047866, as well as funding from the Broad Institute and the Department of Defense. This work was conducted with support from Harvard Catalyst | The Harvard Clinical and Translational Science Center (National Center for Research Resources and National Center for Advancing Translational Sciences, NIH grant UL1-TROO1102), and financial contributions from Harvard University and its affiliated academic health care centers. The contents do not necessarily represent the views of the U.S. Department of Veterans Affairs (VA), the U.S. government, Harvard Catalyst, Harvard University and its affiliated academic healthcare centers, or the National Institutes of Health.

\section{Compliance with Ethical Standards:}

Conflict of Interest: Dr. Ubel is a consultant for Humana. Dr. Green receives compensation for speaking or consultation from AIA, GenePeeks, Helix, Illumina, Ohana, Prudential, and Veritas, and is co-founder, advisor, and equity holder in Genome Medical, Inc. The other authors declare that they do not have a conflict of interest.

\section{REFERENCES}

1. Biesecker LG, Green RC. Diagnostic clinical genome and exome sequencing. N Engl J Med. 2014;370(25):2418-25.

2. Gagan J, Van Allen EM. Next-generation sequencing to guide cancer therapy. Genome Med. 2015;7(1):80.

3. Yang Y, Muzny DM, Xia F, et al. Molecular findings among patients referred for clinical whole-exome sequencing. JAMA. 2014;312(18):1870-9.

4. Cuckle H, Benn P, Pergament E. Cell-free DNA screening for fetal aneuploidy as a clinical service. Clin Biochem. 2015;48(15):932-41.

5. Beaudet AL. Using fetal cells for prenatal diagnosis: History and recent progress. Am J Med Genet C Semin Med Genet. 2016;172(2):123-7.

6. Brison N, Van Den Bogaert $\mathbf{K}$, Dehaspe $\mathbf{L}$, et al. Accuracy and clinical value of maternal incidental findings during noninvasive prenatal testing for fetal aneuploidies. Genet Med. 2017;19(3):306-13.

7. Clinical utility of genetic and genomic services: a position statement of the American College of Medical Genetics and Genomics. Genet Med. 2015; 17(6):505-7.

8. Grosse SD, Rogowski WH, Ross LF, Cornel MC, Dondorp WJ, Khoury MJ. Population screening for genetic disorders in the 21st century: evidence, economics, and ethics. Public Health Genomics. 2010;13(2):106-15.

9. Hampel H. Genetic counseling and cascade genetic testing in Lynch syndrome. Fam. Cancer. 2016;15(3):423-7.

10. Linderman MD, Nielsen DE, Green RC. Personal genome sequencing in ostensibly healthy individuals and the peopleSeq consortium. J Pers Med. 2016;6(2):14.

11. Peplow M. The 100,000 Genomes Project. BMJ. 2016;353:i1757.

12. Precision Medicine Initiative Working Group. The Precision Medicine Initiative Cohort Program - Building a Research Foundation for 21st Century Medicine. 2015. http://www.nih.gov/sites/default/files/research-training/initiatives/pmi/pmi-working-group-report-20150917-2. pdf. Accessed December 13, 2017.

13. Carey DJ, Fetterolf SN, Davis FD, et al. The Geisinger MyCode community health initiative: an electronic health record-linked biobank for precision medicine research. Genet. Med. 2016;18(9):906-13.

14. Gaziano JM, Concato J, Brophy M, et al. Million Veteran Program: a mega-biobank to study genetic influences on health and disease. J Clin Epidemiol. 2016;70:214-23.

15. Green RC, Goddard KA, Jarvik GP, et al. Clinical sequencing exploratory research consortium: accelerating evidence-based practice of genomic medicine. Am. J. Hum. Genet. 2016.

16. Ball MP, Bobe JR, Chou MF, et al. Harvard Personal Genome Project: lessons from participatory public research. Genome Med. 2014;6(2):10.
17. Green RC, Berg JS, Grody WW, et al. ACMG recommendations for reporting of incidental findings in clinical exome and genome sequencing. Genet Med. 2013;15(7):565-74.

18. Kalia SS, Adelman $\mathbf{K}$, Bale SJ, et al. Recommendations for reporting of secondary findings in clinical exome and genome sequencing, 2016 update (ACMG SF v2.0): a policy statement of the American College of Medical Genetics and Genomics. Genet Med. 2017;19(2):249-55.

19. Christensen KD, Vassy JL, Jamal L, et al. Are physicians prepared for whole genome sequencing? A qualitative analysis. Clin Genet. 2016;89(2):228-34.

20. Gray Sw, Park ER, Najita J, et al. Oncologists' and cancer patients' views on whole-exome sequencing and incidental findings: results from the CanSeq study. Genet Med. 2016;18(10):1011-9.

21. Dobson A, El-Gamil A, Pal S, Heath S, DaVanzo JE. Projecting the Supply and Demand for Certified Genetic Counselors: A Workforce Study. Vienna, VA: Dobson DaVanzo \& Associates, September 7, 2016.

22. Zhang H, Yu J, Ming Q, Bao L, Wu B-L, Li P. On the globalization and standardization of medical genetics and genomics as clinical and laboratory specialties. N Am J Med Sci (Boston). 2014;7(4):194-8.

23. Lewis KL, Hooker GW, Connors PD, et al. Participant use and communication of findings from exome sequencing: a mixed-methods study. Genet Med. 2016;18(6):577-83.

24. Evans JP, Powell BC, Berg JS. Finding the rare pathogenic variants in a human genome. JAMA. 2017;317(18):1904-5.

25. McLaughlin HM, Ceyhan-Birsoy O, Christensen KD, et al. A systematic approach to the reporting of medically relevant findings from whole genome sequencing. BMC Med Genet. 2014;15:134.

26. Lek M, Karczewski KJ, Minikel EV, et al. Analysis of protein-coding genetic variation in 60,706 humans. Nature. 2016;536(7616):285-91.

27. Vassy JL, Christensen KD, Schonman EF, et al. The impact of wholegenome sequencing on the primary care and outcomes of healthy adult patients: a pilot randomized trial. Ann Intern Med. 2017;167(3):159-69.

28. Richards S, Aziz N, Bale S, et al. Standards and guidelines for the interpretation of sequence variants: a joint consensus recommendation of the American College of Medical Genetics and Genomics and the Association for Molecular Pathology. Genet Med. 2015; 17(5):405-24.

29. Nambot S, Thevenon J, Kuentz P, et al. Clinical whole-exome sequencing for the diagnosis of rare disorders with congenital anomalies and/or intellectual disability: substantial interest of prospective annual reanalysis. Genet Med. 2017 Nov 2. https://doi.org/10.1038/gim.2017. 162.

30. Vassy JL, Korf BR, Green RC. How to know when physicians are ready for genomic medicine. Sci Transl Med. 2015;7(287):287fs19-fs19.

31. Clark D, Kowal S. Communicating genomic risk in primary health care: challenges and opportunities for providers. Med Care. 2014;52(10):933-4.

32. Vassy JL, Lautenbach DM, McLaughlin HM, et al. The MedSeq Project: a randomized trial of integrating whole genome sequencing into clinical medicine. Trials. 2014;15(1):85.

33. Vassy JL, Christensen KD, Slashinski MJ, et al. "Someday it will be the norm": Physician perspectives on the utility of genome sequencing for patient care in the MedSeq Project. Per Med. 2015; 12(1):23-32.

34. Vassy JL, McLaughlin HM, MacRae CA, et al. A one-page summary report of genome sequencing for the healthy adult. Public Health Genomics. 2015;8(2):123-9.

35. Kong SW, Lee I-H, Leshchiner I, et al. Summarizing polygenic risks for complex diseases in a clinical whole-genome report. Genet Med. 2015; 17(7):536-44.

36. United States Surgeon General. My Family Health Portrait: A Tool from the Surgeon General. 2009. https://familyhistory.hhs.gov/FHH/html/ index.html. Accessed December 13, 2017.

37. Hsieh HF, Shannon SE. Three approaches to qualitative content analysis. Qual Health Res. 2005; 15(9):1277-88.

38. Carroll JC, Makuwaza T, Manca DP, et al. Primary care providers' experiences with and perceptions of personalized genomic medicine. Can Fam Phys. 2016;62(10):e626-e35.

39. Selkirk CG, Weissman SM, Anderson A, Hulick PJ. Physicians' preparedness for integration of genomic and pharmacogenetic testing into practice within a major healthcare system. Genet Test Mol Biomarkers. 2013;17(3):219-25.

40. Korf BR. Genomic medicine: educational challenges. Mol Genet Genomic Med. 2013;1(3):119-22.

41. Najafzadeh M, Davis JC, Joshi P, Marra C. Barriers for integrating personalized medicine into clinical practice: a qualitative analysis. Am J Med Genet A. 2013;161A(4):758-63. 
42. Nippert I, Harris HJ, Julian-Reynier C, et al. Confidence of primary care physicians in their ability to carry out basic medical genetic tasks-a European survey in five countries-Part 1. J Commun Genet. 2011;2(1):1-11.

43. Manolio TA, Murray MF. The growing role of professional societies in educating clinicians in genomics. Genet Med. 2014;16(8):571-2.

44. Feero WG, Manolio TA, Khoury MJ. Translational research is a key to nongeneticist physicians' genomics education. Genet Med. 2014;16(12):871-3.

45. Demmer LA, Waggoner DJ. Professional medical education and genomics. Annu Rev Genomics Hum Genet. 2014;15:507-16.

46. Blazer KR, Christie C, Uman G, Weitzel JN. Impact of web-based case conferencing on cancer genetics training outcomes for community-based clinicians. J Cancer Educ. 2012;27(2):217-25.

47. Carroll JC, Wilson BJ, Allanson J, et al. GenetiKit: a randomized controlled trial to enhance delivery of genetics services by family physicians. Fam Pract. 2011;28(6):615-23.

48. Korf BR, Berry AB, Limson M, et al. Framework for development of physician competencies in genomic medicine: report of the Competencies Working Group of the Inter-Society Coordinating Committee for Physician Education in Genomics. Genet Med. 2014;16(11):804-9.

49. Sharp RR, Goldlust ME, Eng C. Addressing gaps in physician education using personal genomic testing. Genet Med. 2011;13(8):750-1.

50. NHS Health Education England. Introducing Health Education England's Genomics Education Programme. https://www.genomicseducation.hee. nhs.uk/images/publications/NHS_Expo_GEP_Leaflet_final.pdf. Accessed December 13, 2017.

51. Paul J, Metcalfe S, Stirling L, Wilson B, Hodgson J. Analyzing communication in genetic consultations-a systematic review. Patient Educ Couns. 2015;98(1):15-33.

52. Arora NS, Davis JK, Kirby C, et al. Communication challenges for nongeneticist physicians relaying clinical genomic results. Pers Med. 2016; 14(5):423-431.

53. Sarangi S. The language of likelihood in genetic-counseling discourse. J Lang Soc Psychol. 2002;21(1):7-31.
54. O'Doherty K. Risk communication in genetic counselling. Theory Psychol. 2006; 16(2):225-56.

55. O'Doherty KC, Navarro DJ, Crabb SH. A qualitative approach to the study of causal reasoning in natural language. Theory Psychol. 2009;19(4):475-500.

56. Thomassen G, Sarangi S. Evidence-based familial risk explanations in cancer genetic counselling. Health Risk Soc. 2012;14(7-8):607-26.

57. Lehtinen E, Kääriäinen H. Doctor's expertise and managing discrepant information from other sources in genetic counseling: a conversation analytic perspective. J Genet Couns. 2005;14(6):435-51.

58. Lehtinen E. Hedging, knowledge and interaction: Doctors' and clients' talk about medical information and client experiences in genetic counseling. Patient Educ Couns. 2013;92(1):31-7.

59. Scheuner MT, Edelen MO, Hilborne LH, Lubin IM. Effective communication of molecular genetic test results to primary care providers. Genet Med. 2013;15(6):444-9.

60. Williams JL, Rahm AK, Stuckey H, et al. Enhancing genomic laboratory reports: a qualitative analysis of provider review. Am J Med Genet A. 2016;170A(5):1134-41.

61. Shirts BH, Salama JS, Aronson SJ, et al. CSER and eMERGE: current and potential state of the display of genetic information in the electronic health record. J Am Med Inform Assoc. 2015;22(6): 1231-42.

62. McGuire AL, Burke W. An unwelcome side effect of direct-to-consumer personal genome testing: raiding the medical commons. JAMA. 2008;300(22):2669-71.

63. Korngiebel DM, Fullerton SM, Burke w. Patient safety in genomic medicine: an exploratory study. Genet Med. 2016;18(11):1136-42.

64. Deyo RA. Cascade effects of medical technology. Annu Rev Public Health 2002;23:23-44.

65. Vassy JL, Bates DW, Murray MF. Appropriateness: a key to enabling the use of genomics in clinical practice? Am J Med. 2016;129(6):551-3.

66. Overby CL, Kohane I, Kannry JL, et al. Opportunities for genomic clinical decision support interventions. Genet Med. 2013;15(10):817-23. 